\title{
Theoriefreie Klassifikation psychischer Störungen
}

\author{
Atheoretical Classification of Psychiatric Disorders
}

Autoren

Pro: Hans Förstl

Kontra: Paul Hoff

Bibliografie

DOI 10.1055/s-0028-1090203

Psychiat Prax 2009; 36: 55-57

(c) Georg Thieme Verlag KG

Stuttgart · New York .

ISSN 0303-4259

Korrespondenzadressen

Prof, Dr. med. Hans Förstl

Klinik und Poliklinik für Psych-

iatrie und Psychotherapie,

TU München

Ismaningerstraße 22

81675 München

hans.foerstl@|rz.tu-

muenchen.de

\section{Prof. Dr. med. Dr. phil.}

\section{Paul Hoff}

Psychiatrische Universitätsklinik

Zürich, Klinik für Soziale

Psychiatrie und Allgemein-

psychiatrie ZH West

Lenggstraße 31

Postfach 1931

8032 Zürich, Schweiz

paul.hoff@puk.zh.ch

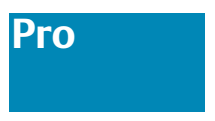

Für den Zweck dieser Diskussion ist hier die intellektuell radikale These zu vertreten, dass psychische Störungen ohne vorherige Festlegungen einzuteilen sind. Theoretische Grundannahmen sollten zuvor nicht getroffen werden.

Was kann man an der „Theorie“ in der Psychiatrie aussetzen? Die Konnotation des „Theorie“-Begriffs legt eher akademische Auseinandersetzungen und philologische Erstarrung nahe, als ein neugieriges Schauen und dynamisches Entwickeln im eigentlichen Wortsinn. Diese Theorie scheint der Praxis geradezu entgegen gesetzt. Bei den rein menschengemachten Disziplinen wie Philologie und Juristerei mag man sich mit Theorien begnügen. Die „richtigen“ Wissenschaften bohren sich in ein tieferes Verständnis der Natur und des Menschen hinein und lassen ihre Theorien ständig hinter sich. Jeder Arzt lernt mithilfe seiner Patienten ständig dazu und verändert seine Sichtweise, seine Theorie. Temporäre diagnostische Konstrukte, die eine tiefere Bedeutung bis hin zur Ätiologie suggerieren, nutzen allenfalls zur Tablettenwahl und zur Leistungsabrechnung [1].

Also dienen die Theorien recht profanen Zwecken, die ihren Schöpfern erheblich gegen den Strich gingen. Kraepelin, dem Agnaten der wissenschaftlichen Psychiatrie des 20. Jahrhunderts, ging es tatsächlich um besondere Werte. Sein Minderwertigkeitsempfinden gegenüber den anderen Fächern der Medizin, die bereits naturwissenschaftlich, also damals durch die Pathologie unterfüttert waren und die klare mechanistische Vorstellungen über Entstehung und Behandlung ihrer Krankheiten verkündeten, führte zu einer recht entschlossenen theoretischen Aufrüstung, nachzulesen in den vielen Auflagen seines Lehrbuchs. Instinktsicher setzte er auch auf die richtigen Forschungsgebiete, allerdings hinkten die empirischen Arbeiten dem theoretischen Entwurf hinterher.
Als Reaktion auf die romantische Antipsychiatrie der 60er- und 70er-Jahre fasste Klerman [2] das Programm des Kraepelinismus nochmals neu zusammen: Psychiatrie sei demnach ein medizinisches Fach; sie benutze moderne wissenschaftliche Methoden; zwischen gesund und psychisch krank gebe es eine klare Grenze; auch zwischen den einzelnen psychischen Krankheiten gebe es klare Grenzen; ... Diagnostik und Klassifikation seien ein erklärtes Ziel psychiatrischer Forschung; sie sollten akademisch gelehrt und nicht ständig in Zweifel gezogen werden. Eine der Klassifikation dienende Theorie war also essenzieller Stützpfeiler einer wissenschaftlichen Psychiatrie nach Kraepelin.

Nun ist aber die Antipsychiatrie mausetot. Selten waren Medizinstudenten so positiv eingestellt, neugierig und gleichzeitig so pragmatisch wie heute. Dieser moderne Pragmatismus wäre auch den Fachvertretern zu empfehlen. Zu einer anwendungsbezogenen Auseinandersetzung mit dem Fach Psychiatrie gehört zugegebenermaßen auch das zügige Durchdeklinieren von Diagnosekriterien als Mittel zum Zweck. Die junge Generation der Kollegen blickt aber durch, sie durchschaut die Theorien bis auf ihren aktuellen naturund sozialwissenschaftlichen Grund.

Geschichte und Gegenwart der Psychiatrie belegen vielfach, dass es unzeitgemäß und ganz ungeschickt wäre, theoretische Ansichten noch in Marmor zu meisseln. Im Folgenden sollen einige warnende Beispiele die Vergänglichkeit, Unvollständigkeit, Sinnleere oder Beeinflussbarkeit psychiatrischer Theoriebildung illustrieren.

Zu den historischen Exempeln zählt das Arbeitsprogramm, dass sich Karl Philipp Moritz im 18. Jahrhundert gab: In der ersten psychologischnervenärztlichen Zeitschrift „Gnothi Sauton“ wollte er erst ein paar Jahre lang Material sammeln (Fallgeschichten, Selbstbeschreibungen, theoretische Vorüberlegungen etc.), um dann eine schlüssige Theorie des Geistes und seiner 
Störungen zu formulieren. Das liegt über 200 Jahre zurück, und Moritz hat es nicht geschafft [3].

Die sinnleere Verselbstständigung biomathematischer Methoden ist ein Beispiel, das weniger weit zurückliegt. Mit dem Einzug von Computern und Statistikpaketen erblühten Ende des 20. Jahrhunderts in Cluster und Faktoren aufgefaltete Syndrome, die dem unbewaffneten Auge des Klinikers sonst verborgen geblieben wären und vielfach frei von klinischer Bedeutung blieben [4]. Viele von uns psychiatrischen Forschern sind ihrem Reiz dennoch zeitweise erlegen.

Mit dem aktuellen Paradigmenwechsel hin zur Welt der Molekulargenetik, etwa mit der Definition von „Endophänotypen“, werden frühere Theoriebildungen von Grund auf infrage gestellt. Beim Versuch, die langen und verschlungenen Wege von den Genen über die Genprodukte und deren potenzielle Interaktionen unter Umwelteinflüssen bis hin zum Verhalten und Erleben der „Träger“ nachzuzeichnen, lösen sich die konventionellen Krankheitsbegriffe auf [5].

Dass psychiatrische Theorien nicht nur wissenschaftlich erarbeitet und begründet sind, sondern auch erheblich von gesellschaftlichen und ökonomischen Rahmenbedingungen beeinflusst werden, soll nicht verschwiegen werden. Theorien implizieren zum Beispiel Therapieoptionen und damit Umsatzchancen. Hier zu das Beispiel der affektiven Störungen: Das Kommen und Gehen von Krankheitskonzepten wurde und wird nicht nur von Philosophie und Zeitgeist („Melancholie“), neuen Beobachtungen (z.B. von Falret und Baillarger ab 1830 zu den zyklisch verlaufenden affektiven Erkrankungen), klaren theoretischen Setzungen wie Kraepelins Dichotomie [6], sondern auch durch die Industrie und ihre Produkte beeinflusst (bipolar boom).

Das warnende Beispiel der klassischen Psychoanalyse, bei der kühne Theorie den Mangel an Substanz dauerhaft überschattet, lasse ich an dieser Stelle ganz unerwähnt.

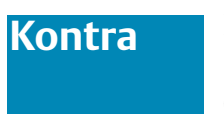

Meine Argumentation gliedert sich in drei Abschnitte: In Anbetracht der unübersichtlichen Geschichte psychiatrischer Konzepte, vor allem solcher zur Ätiologie und zur Klassifikation, ist es nur zu verständlich, dass immer wieder die Forderung nach „weniger Theorie“" erhoben wurde (I). Nicht nachvollziehbar ist hingegen, wenn operationalisierte Diagnosemanuale (ICD-10, DSM-IV) als Beispiele einer realisierten „Theoriefreiheit“ in der psychiatrischen Diagnostik gelobt werden (II). Der Psychopathologie in erweiterter Form könnte bei der zukünftigen Entwicklung dieses Feldes eine wichtige Rolle zukommen (III).

\section{(I) Eine verständliche Forderung}

In den vergangenen 200 Jahren entstand eine unübersichtlich große und heterogene Gruppe von psychiatrischen Krankheitsmodellen, die alle - wenn auch in unterschiedlicher Schärfe beanspruchten, den richtigen Weg für Diagnostik, Therapie und Forschung zu weisen. Um so verständlicher ist es, dass ab den 60er-Jahren des 20. Jahrhunderts die Notwendigkeit großer und internationaler empirischer Studien zur Evaluation neuer diagnostischer (z.B. Bildgebung) und therapeutischer Verfahren (z.B. Psychopharmaka) zu der Forderung führte, eine reliable und für verschiedene Schulen akzeptable psychiatrische Terminologie zu schaffen. Anders ausgedrückt: Eine zunehmend evidenzbasierte Psychiatrie erzwang eine vermehrte nationale und internationale wissenschaftliche Kommunikation, wobei die einzelnen Begriffe möglichst nah am Beobachtbaren und fern von ätiologischen Hypothesen, eben theoriearm, sein sollten.

\section{(II) Operationale Diagnostik als Beispiel für "Theoriearmut"?}

Speziell die Begriffe der psychiatrischen Diagnostik sollten unabhängig vom sprachlichen und kulturellen Hintergrund des Untersuchers möglichst eindeutig definiert und operationalisiert sein. Im Kontext der mit diesem Ziel entwickelten Diagnosemanuale ICD-10 und DSM-IV brachte es nun der Terminus „Theoriefreiheit“ zu großer Verbreitung, obwohl er von Beginn an unglücklich gewählt war: Es konnte in Wirklichkeit nie von der Absenz jeglicher Theorie die Rede sein. Gemeint waren vielmehr die folgenden, vor allem aus Sicht der empirischen Forschung bedeutsamen Ziele:

- Fokussierung auf beobachtbare, beschreibbare, quantifizierbare psychopathologische Phänomene („deskriptiver Ansatz“)

- Erkennen und Vermeiden impliziter theoretischer Vorannahmen zur Ätiologie und Pathogenese (irreführend als „Theoriefreiheit" bezeichnet)

- Akzeptanz bei verschiedenen ätiologischen Schulen ( „größter gemeinsamer Nenner“)

Doch zeigt sich auch hier die Janusköpfigkeit vermeintlich einfacher und klarer wissenschaftlicher Regeln in einem so hochkomplexen Umfeld wie der psychiatrischen Diagnostik: Wendet man nämlich diese Regeln, i.e. operationale Algorithmen, unkritisch an, so erreicht man im schlimmsten Fall das genaue Gegenteil dessen, was intendiert war: Die Konzentration auf das Beobachtbare wird dann jedes nicht in den Kriterienlisten erfasste oder erfassbare psychopathologische Phänomen unterschätzen, verkennen oder ignorieren. Auf jeden Fall wird es für unwissenschaftlich erklärt. So aber führt die durchaus berechtigte Sorge vor „zu viel Theorie“ erst recht zu dogmatischer Verkürzung, hier auf dem theoretischen (!) Hintergrund von Positivismus und logischem Empirismus. Anders formuliert: Aus der Bevorzugung der für Objektivierung und Quantifizierung attraktiven 3.-Person-Perspektive („Er hört Stimmen“) kann unbesehen eine Missachtung der am subjektiven Erleben orientierten 1.-Person-Perspektive („Ich höre Stimmen“) sowie der 2.-Person-Perspektive werden, die den intersubjektiven Charakter des diagnostischen Prozesses betont („Er/Sie schildert mir, dass er/ sie Stimmen hört“). Auch der Umstand, dass jede medizinische Diagnose, auch die psychiatrische, nicht nur eine faktenbezogene, sondern auch eine Wertdimension hat, wird von der operationalisierten Diagnostik unterschätzt [7].

Ergo: Die operationale Diagnostik hat zweifellos zu einem transparenteren, reliableren und - in einem bestimmten Sinn, nämlich ätiologisch - auch theorieärmeren Vorgehen geführt. Dies ist ein bemerkenswerter Fortschritt. Aber auch dieser Ansatz hat seine Grenzen, und ganz bestimmt ist er nicht theoriefrei.

\section{(III) Über die zukünftige Rolle der Psychopathologie}

Die Psychopathologie ist - nicht erst seit gestern - markant in die Defensive geraten [8]. Vor allem ihr Anspruch, Grundlagenwissenschaft der Psychiatrie zu sein [9], wird nachhaltig bestritten, ja mitunter wird gar ihre Abschaffung bzw. Ersetzung durch neurowissenschaftlich fundierte Konzepte prognostiziert. Doch wird gerade in unserem jetzigen Kontext eine mögliche zukünftige Ausrichtung und Aufwertung der Psychopathologie erkennbar, könnte sie doch eine kritische und gleichwohl konstruktive Grundhaltung gegenüber scheinbar einfachen Gesamterklärun- 
gen, die in Wirklichkeit Vorurteile sind, in die diagnostische Praxis und den wissenschaftlichen Diskurs tragen [10].

Also: Nicht Theorie als solche ist von Übel, sondern theoretische Vorurteile. Karl Jaspers' diesbezügliches Monitum ist aktueller denn je und gilt für alle psychiatrischen Konzepte in gleicher Weise [11]:

„Wo ein theoretisches Vorurteil herrscht, wird die Auffassung der Tatbestände befangen. Man sieht die Befunde immer schon im Schema der Theorie. Was für sie gilt und sie bestätigt, das interessiert. Was keinen Bezug auf sie hat, das wird überhaupt nicht wahrgenommen. ... Es ist daher ständig unsere Aufgabe, von theoretischen Vorurteilen, die jederzeit in uns wirksam sind, absehen zu lernen, uns zu üben, rein die Befunde aufzufassen." ([12], S. 15)

Aktuell beobachten wir ein wachsendes Spannungsfeld zwischen einer betont deskriptiv-,,theoriearmen“ Diagnostik einerseits (z. B. ICD-10) und „theoriereichen“ Krankheitsmodellen andererseits, die weitgehend unabhängig von der psychopathologischen Ebene entstanden sind, etwa neurowissenschaftliche Konzepte der Schizophrenie als Hirnentwicklungsstörung [13] oder als Folge gestörter basaler Informationsverarbeitung [14]. Ein erweitertes Verständnis von Psychopathologie könnte hier als verbindende Klammer dienen, was freilich ein sehr hoher Anspruch ist, den es erst einmal einzulösen gilt. Denn nicht nur um die operationale Deskription und Verknüpfung von Einzelphänomenen ginge es dann, sondern auch um eine „offene“ Deskription, die subjektive und hermeneutisch-biographische Momente - also die 1.- und 2.-Person-Perspektive - aktiv einbezieht. Dies wiederum setzt die Verankerung in der psychiatrischen Ideengeschichte ebenso voraus wie die Bereitschaft, vorschnellen Antworten auf grundlegende Fragen unseres Faches (Leib-Seele-, Subjekt-Objekt-Frage, Personalität und Verantwortung) eine Absage zu erteilen.

Wir sollten auf dem Boden einer aufgewerteten Psychopathologie die unfruchtbare Polarisierung „theoriereich“ vs. „theoriearm“ ",theoriefrei“ hinter uns lassen. Nur dann nämlich könnte das für die künftige Identität der Psychiatrie schädliche „postmoderne" Nebeneinander deskriptiv-operationaler, neurobiologischer, subjektiv-qualitativer und sozialwissenschaftlicher Befunde durch eine sinnvolle Integration aller relevanten Perspektiven abgelöst werden. In diese Richtung denken erfreulicherweise auch die mit der Planung von ICD-11 und DSM-V beschäftigten Expertengremien. Jüngst erschienene Bücher zur Psychopathologie und vor allem zu deren phänomenologischer Perspektive belegen überzeugend, dass „Theorie“ für die empirische
Forschung eben nicht hinderlich ist, sondern sie kritisch begleitet und anregt [15-17]. Prägnanter: Was wir brauchen in der Psychiatrie, ist keine „Theoriephobie“, sondern reflektierte und damit undogmatische Theorie.

\section{Literatur}

1 Cooper R. Psychiatry and Philosophy of Science. Stocksfield: Acumen Publ, 2007

2 Klerman GL. The evolution of a scientific nosology. In: Schizophrenia. Science and Practice, 1978

3 Förstl H, Angermeyer M, Howard R. Karl Philipp Moritz' Journal of Empirical Psychology (1783-1793): an analysis of 124 case reports. Psychol Med 1991; 21: 299-304

4 Kendler KS, Karkowski LM, Walsh D. The structure of psychosis - latent class analysis of probands from the Roscommmon family study. Arch Gen Psychiat 1998; 55: 492-99

5 Braff DL, Freedman R, Schork NJ et al. Deconstructing schizophrenia: an overview of the use of endophenotypes in order to understand a complex disorder. Schizophrenia Bull 2007; 33: 21-32

6 Angst J, Marneros A. Bipolarity from ancient to modern times: conception, birth and rebirth. J Affect Dis 2001; 67: 3-19

7 Fulford KWM, Thornton T, Graham G. Values, Ethics, and Mental Health. In: Part IV of Fulford KWM, Thornton T, Graham G eds. The Oxford Textbook of Philosophy and Psychiatry. Oxford: Oxford University Press, 2006

8 Janzarik W. Die Krise der Psychopathologie. Nervenarzt 1976; 47: 73-80

9 Janzarik W. Psychopathologie als Grundlagenwissenschaft. Stuttgart: Enke, 1979

10 Hoff $P$. Über die zukünftige Rolle der Psychopathologie: Grundlagenoder Hilfswissenschaft? In: Fuchs T, Vogeley K, Heinze M, Hrsg. Subjektivität und Gehirn. Lengerich: Pabst Science Publishers, 2007: 195-209

11 Hoff $P$. Erkenntnistheoretische Vorurteile in der Psychiatrie - eine kritische Reflexion 75 Jahre nach Karl Jaspers' „Allgemeiner Psychopathologie“. Fundamenta Psychiatrica 1989; 3: 141-150

12 Jaspers K. Allgemeine Psychopathologie. Berlin: Springer, 1913 (zitiert ist die 4., völlig neu bearbeitete Aufl. Heidelberg: Springer, 1946

13 Bogerts B, Lieberman J. Neuropathology in the study of psychiatric disease. In: Costa e Silva ACJ, Nadleson CC, eds. International review of psychiatry. Washington DC: American Psychiatric Press, 1993

14 Möller HJ, Kasper S. Die Rolle der Kognition in der Therapie schizophrener Störungen. Wiesbaden: Deutscher Universitätsverlag, 2000

15 Gallagher S, Zahavi D. The Phenomenological Mind. An Introduction to Philosophy of Mind and Cognitive Science. London: Routledge, 2008

16 Kendler KS, Parnas J eds. Philosophical Issues in Psychiatry: Explanation, Phenomenology, and Nosology. Baltimore: Johns Hopkins University Press, 2008

17 Thompson E. Mind in Life. Biology, Phenomenology, and the Sciences of Mind. Cambridge: The Belknap Press of Harvard University Press, 2007 